\title{
Performance Evaluation of Virtual Traffic Light (VTL) Algorithm Vs Traffic Light System (TLS) in SUMO
}

\author{
Tin Maung Wynn \\ Department of Information Technology, \\ Technological University(Thanlyin) \\ Yangon, Myanmar
}

\author{
Wah Wah Kyaw \\ Department of Electronic Engineering, \\ Technological University(Toungoo) \\ Toungoo, Myanmar
}

\begin{abstract}
In future, all the vehicles will be the connected vehicles and they can communicate with each other. At that time, physical traffic light systems will be replaced with Virtual Traffic Light systems. The Virtual Traffic Light (VTL) is a self-organizing traffic control system without requiring road infrastructures. However, the major weakness of the existing VTL allows only one vehicle crossing the intersection at a time and considers only straightforward direction of vehicles. This paper implements a VTL algorithm for road intersections on Simulation of Urban Mobility (SUMO) that allows more vehicles to cross the intersection without collisions and considers the vehicle's other directions such as turn left or turn right rather than go straight. For performance evaluation, the $\mathrm{CO}_{2}$ emission of vehicles applying the proposed VTL is compared to that of physical traffic light system (TLS) in SUMO. According to comparison result, the average $\mathrm{CO}_{2}$ emission of vehicles applying the proposed VTL algorithm significantly decreases compared to traffic light system in SUMO.
\end{abstract}

Keywords: ITS, VTL, TLS, SUMO

\section{INTRODUCTION}

As Smart Vehicles (SVs) become more common, the traffic control strategies that are currently used for human drivers will likely have to be re-thought. An important example is that of intersection management (IM): in a typical traffic light setting, vehicles are not allowed to cross the intersection if the light corresponding to their lane is red, even in the absence of conflicting vehicles. This often results in many vehicles unnecessarily idling, and can significantly increase their $\mathrm{CO}_{2}$ emissions. Fully leveraging the capacities of smart vehicles, various autonomous IM (AIM) techniques that do not require a signal-based approach have been proposed in [1]. One of the approaches is to implement VTL system for smart vehicles.

Nowadays, traffic lights currently control only a limited number of intersections, and increasing the number of traffic lights is clearly infeasible due to the high cost of deployment and maintenance [2]. To overcome this challenge, the first distributed VTL algorithm for Vehicular Ad-hoc NETworks (VANETs) has been described in [3], where simulation results showed up to $60 \%$ increase in the average flow rate in the reference city of Porto. The adopted algorithm is based on the definition of cluster of vehicles, cluster head, and VTL leader. The vehicles on the same road form a cluster and the one which is nearest to the intersection is the cluster head. The cluster head that is farther from the intersection is then elected as the VTL leader and is responsible for determining the priorities of vehicles and broadcasting the virtual traffic light messages. Once the VTL leader leaves the intersection, a new VTL leader is elected.

The same algorithm has been in many subsequent studies. The distributed VTL algorithms introduced in [2, 4] exchange information between smart vehicles using both broadcast messages for signaling and unicast messages for precedence definition and traffic light decisions. The algorithm has been implemented and tested through low cost IEEE $802.11 \mathrm{p}$ devices, using open source software. But, the distributed VTL algorithms in [4] and [2] are designed with the assumption that they are not able to infer the future movements of each SV and only one SV can cross the intersection at a time, even though there will be no possibility of collision between SVs. For example, two SVs approaching the intersection from opposite directions with the intent to go straight would not need to stop at the intersection.

Although some VTL algorithms were introduced for VANETs, they allow only one vehicle to cross the junction at a time. While a vehicle from one road segment is crossing the junction, the vehicles from the other three road segments cannot cross the junction. Moreover, the VTL algorithms consider only straightforward directions of vehicles.

In this study, a VTL algorithm for road intersection is proposed to optimize the existing VTL algorithm. The proposed VTL algorithm enables more than one vehicle crossing the intersection simultaneously without collision and and considers the vehicle's other directions such as turn left or turn right rather than go straight. The algorithm is intended for intersections where the deployment of real traffic lights is not cost-effective. The algorithm was implemented and tested in SUMO. As a simulation result, the $\mathrm{CO}_{2}$ emission of vehicles using the proposed VTL algorithm is compared with the TLS in SUMO.

The rest of this paper is organized as follows: The proposed VTL algorithm is mentioned in detail in Section 2. In Section 3, the implementation of the proposed VTL algorithm is described and the simulation result and discussion is shown in Section 4. Finally, the conclusion is drawn in Section 5.

\section{THE PROPOSED VIRTUAL TRAFFIC LIGHT ALGORITHM}

The proposed VTL algorithm is based on the firs coming, the first crossing principle for the initial coordination.

\subsection{Assumptions of Original VTL algorithm}

The VTL algorithm knows the position of each vehicle from the SUMO's built-in localization system. By using this information, the VTL algorithm gets the updated positions of vehicles and is able to calculate the priority to cross the intersection. 

concepts:

The VTL algorithm is based on the following key

- Each smart vehicle (SV) is assigned a priority in order to cross the intersection. The priority is calculated based on the distance between $\mathrm{SV}$ and the intersection.

- For each road segment, the SV that is closest to the intersection is denoted as leader and the others as followers. One of the leaders is elected as the intersection leader and it has the priority to cross the intersection.

- If the vehicles are driving in opposite directions or the same road segment with the intersection leader, these vehicles can cross the intersection simultaneously.

- If the vehicles with priorities to cross the intersection have passed the junction, the priority is granted to the vehicles from another road segment.

Figure 1 shows the example of the virtual traffic light, in which the intersection leader, leaders and follower can be seen.

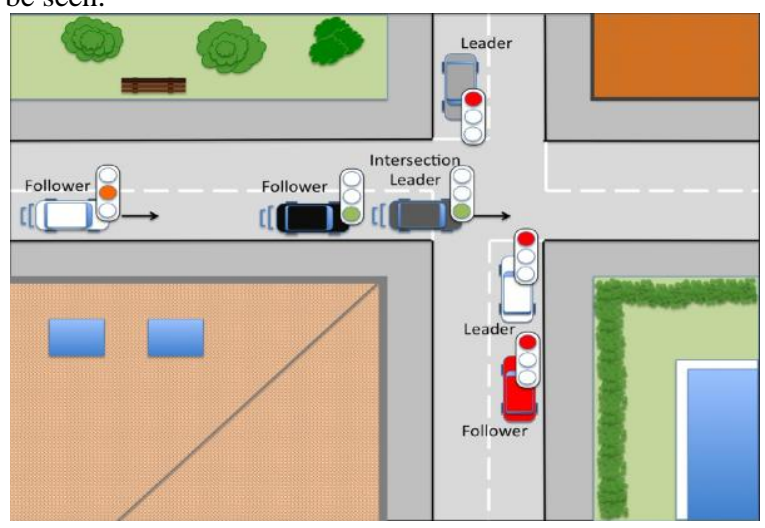

Figure 1. Virtual traffic light example scenario [2]

\subsection{Working Procedure of the proposed}

\section{VTL Algorithm}

In order to implement the proposed VTL algorithm in real world, each smart vehicle must be equipped with a global navigation satellite system such as Global Positioning System (GPS) and short range wireless communication system based on IEEE 802.11p technology. Each SV knows its own position using GPS and broadcasts to neighbour vehicles via short range wireless communication system.

The proposed VTL algorithm starts when the vehicles enter the VTL area. In this study, the VTL area's threshold value is denoted as eighty meters (VTL area's diameter of 160 meters) from the junction because the packet loss rate using IEEE $802.11 \mathrm{p}$ remains lower at distances up to 200 meters when SVs communicate each other. For longer distances, packet loss rate increases. The algorithm executes the following steps.

Step 1) Update the positions of vehicles and calculate the distance from the vehicles to the junction.

Step 2) If the vehicle's distance to the junction is less than eighty meter, set the vehicle's VTL as Orange (Orange VTL) and move to Step 3. Otherwise, return to Step 1.

Step 3) Separate the vehicles based on road segment ID. (There can be at most four vehicles group for a junction.)

Step 4) Sort vehicles on each road segment (NNorth, S-South, W-West and E-East) according to their distance from the junction.
Assign the vehicle that is closest to the junction as the leader and the others as followers.

Step 5) Define two priority zones NS (vehicles from $\mathrm{N}$ and $\mathrm{S}$ road segments) and WE (vehicles from $\mathrm{W}$ and $\mathrm{E}$ road segments).

Step 6) Get leader from each road segment and form a leaders set.

Step 7) Sort the vehicles from the leaders set based on their distance from the junction. Set the first vehicle from the sorted leaders set as the intersection leader. (If all the leaders are equal distances from the junction, the intersection leader is elected based on road segments.)

Step 8) If a vehicle is intersection leader, set the vehicle's VTL as Green (Green VTL) and move to Step 10. Otherwise, move to Step 9.

Step 9) Set the vehicle's VTL as Red (Red VTL) and slow down the vehicle.

Step 10) If the intersection leader is in NS zone, set the vehicles' VTLs in NS as Green (Green VTLs) and define VTL priority as ' $n s$ ', then move to Step 14. Otherwise, move to Step 11.

Step 11) Set the vehicles' VTLs in WE as Green (Green VTLs) and define VTL priority as 'we'. Move to Step 14

Step 12) If the VTL priority is 'ns' and there is no vehicle in NS zone, set the vehicles' VTLs in WE as Green (Green VTLs) and move to Step 14. Otherwise, move to Step 13.

Step 13) If there is no vehicle in WE zone, set the vehicles' VTLs in NS as Green (Green VTLs) and move to Step 14

Step 14) Calculate the priorities of the two vehicles attempting to cross the junction from the opposite directions. (The working procedure to calculate the priority is described in the following).

In order to calculate the priorities, the two conditions, non-collision condition and possible collision condition of two opposite vehicles (SV1 and SV2) are defined as shown in Figure 2 and Figure 3.

\begin{tabular}{|cccc|c|c|}
\hline & Non-collision Condition \\
\hline & SV1 & SV2 & Straight & Straight \\
\hline 3 & Turn-Left & Turn-Left \\
\hline 4 & Turn-Right & Turn-Right \\
\hline 5 & Turn-Right & Straight & Turn-Right
\end{tabular}

Figure 2. Non-collision condition of two opposite vehicles 


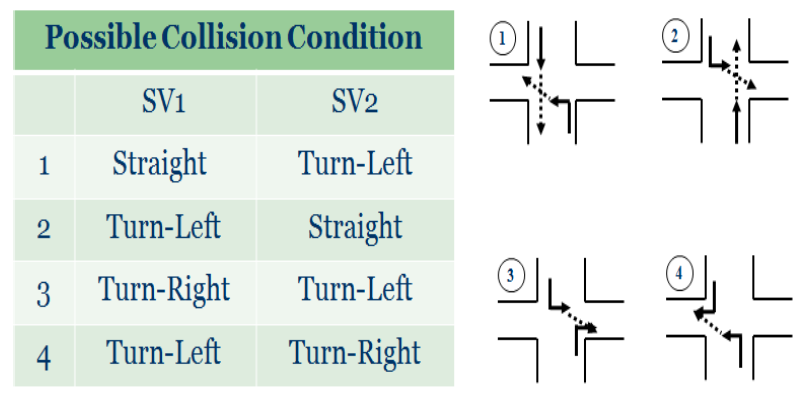

Figure 3. Possible collision condition of two opposite vehicles

Calculation of the priorities of the two vehicles attempting to cross the junction from the opposite directions includes the following steps:

Step 1) Get the two vehicles' signals for directions (Straight, Turn-Left, Turn-Right).

Step 2) If the two vehicles are in the non-collision condition, they can cross the junction simultaneously. Otherwise, move to Step 3.

Step 3) Calculate the distance of the two vehicles to the junction.

Step 4) If one vehicle whose distance to junction is lower than the other, move to Step 5. Otherwise, move to Step 6.

Step 5) Give the priority to the nearest vehicle to the junction and the other vehicle needs to slow down until the prioritized vehicle pass the junction.

Step 6) If the two vehicles are in the same distance to junction and in one of the possible collision conditions, follow the right-of-way rules.

\section{IMPLEMENTATION}

The proposed VTL algorithm was developed using Matlab, SUMO traffic simulator and Traffic Control Interface for Matlab (TraCI4Matlab).

To implement the proposed VTL algorithm in SUMO, the scenario road network file is created using NETEDIT application. In this simulation, a road network with a junction that contains four road segments is created. Then, the route file is created to add vehicles and to define their routes. In this scenario, the proposed VTL algorithm is tested with twelve smart vehicles with their respective directions as in Figure 4(a), and the proposed VTL is represented with vehicle's colour as shown in Figure 4(b).

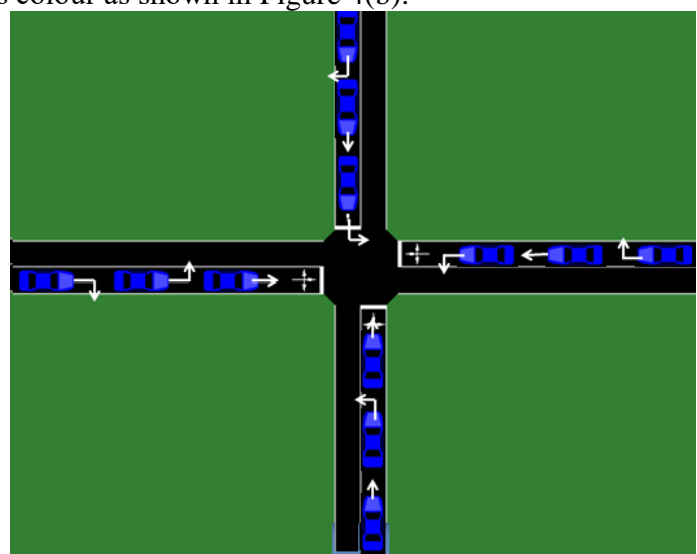

Figure 4(a). Simulation of the proposed VTL with 12 SVs

\subsection{Vehicle's original colour \\ DrangeVTL (enters VTL area) \\ Ded VTL (no priority to cross the junction) \\ Dreen VTL (priority to cross the junction) \\ Figure 4(b). Definition of vehicle's colour in the proposed VTL}

When the simulation starts, the vehicle's colour is set to blue. When a vehicle enters the VTL area, the vehicle's colour is changed from blue to orange (Orange VTL), and then green (Green VTL) or red (Red VTL).

In Figure 5, twelve SVs are approaching the junction from four different road segments (N, S, W and E). SV1, SV4, SV7 and SV10 are leaders of four different road segments and the other eight vehicles will be followers when they enter the VTL area. Then the SVs are sorted on each road segment according to their distance to the junction and the algorithm define two priority zones NS (SV4, SV5, SV6, SV10, SV11, SV12) and WE (SV1, SV2, SV3, SV7, SV8, SV9). Among four leaders, SV10 in $S$ is closest to the junction and it is elected as intersection leader. The algorithm defines VTL priority as ' $n s$ '. The algorithm sets SV10 as Green VTL which means that SV10 can cross the junction and sets the vehicles from NS zone as Green VTL because the intersection leader is in NS zone and calculate the priorities of vehicles. Then, the algorithm sets the vehicles from WE zone as Red VTL as shown in Figure 6. The speed of Red VTL vehicles is decelerated.

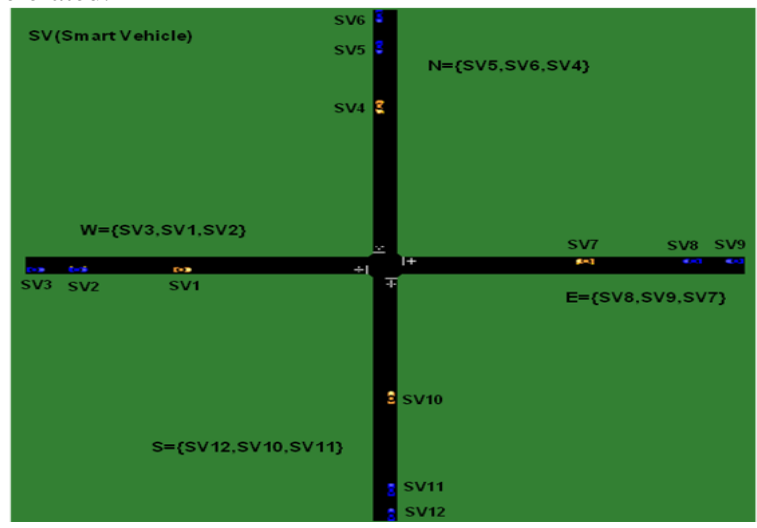

Figure 5. Example scenario of proposed VTL

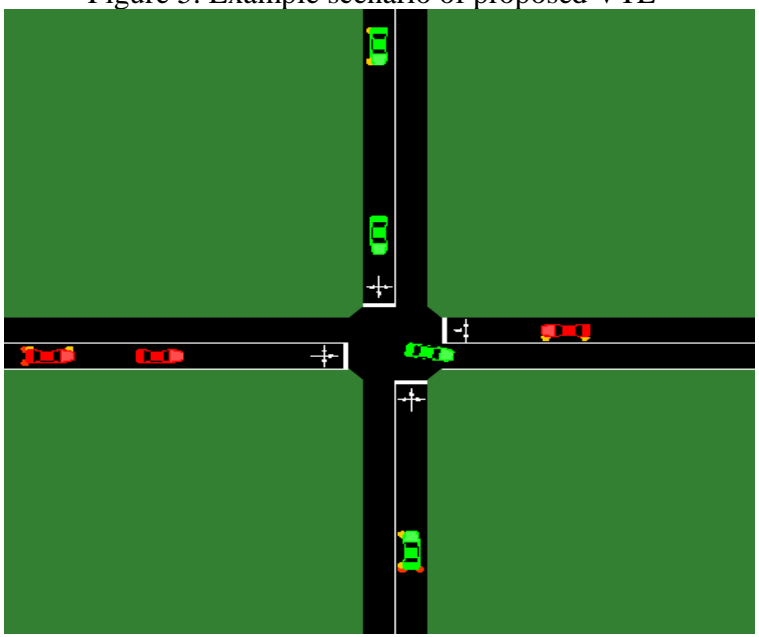

Figure 6. The state of VTL priority in ' $n s$ 
If the VTL priority is 'ns' and there is no vehicle in NS zone, the algorithm sets the vehicles from WE zone as Green VTL as shown in Figure 7, defines VTL priority as 'we' and calculate the priorities of vehicles.

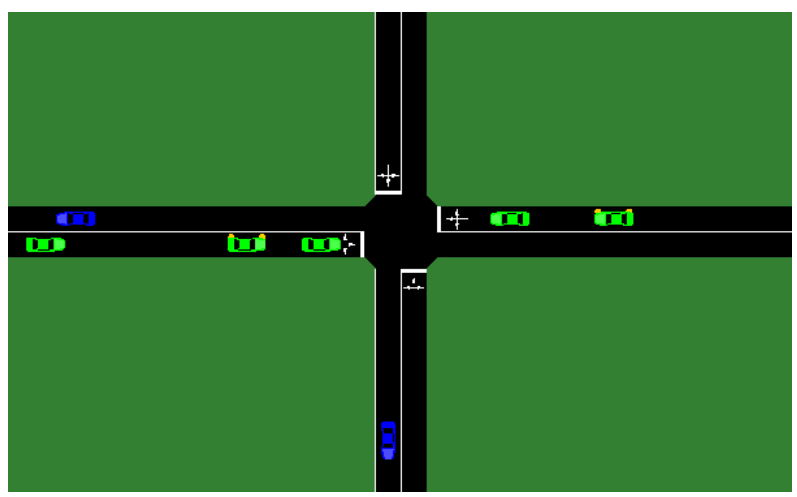

Figure 7. The state of VTL priority in ' $w e$ '

\section{RESULTS AND DISCUSSIONS}

To evaluate the proposed VTL algorithm compared with physical traffic lights, another simulation with twelve vehicles using SUMO's traffic lights (TLS) is implemented and tested as shown in Figure 8. The default green phase length of 33 seconds is used in TLS in SUMO.

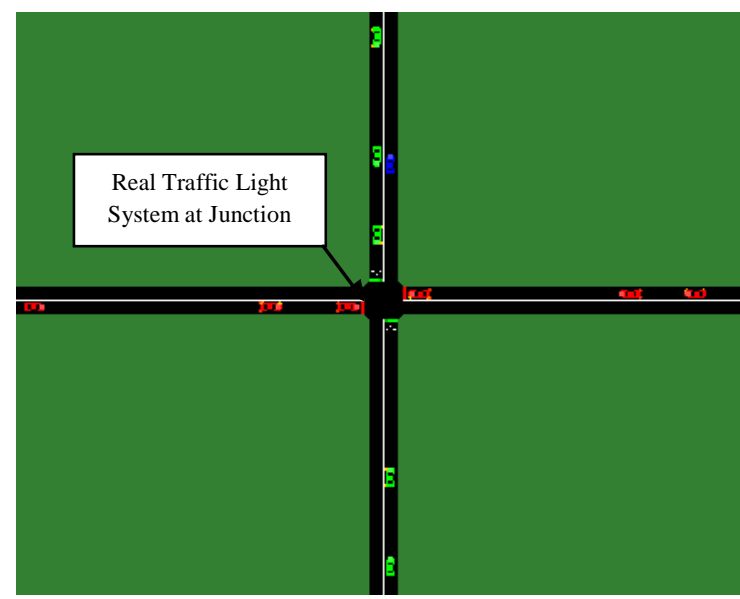

Figure 8. Traffic light simulation with twelve SVs

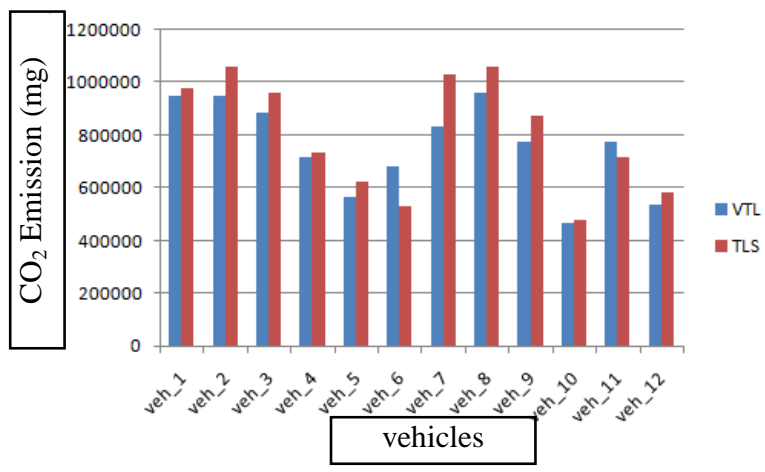

Figure 9. $\mathrm{CO}_{2}$ Emission of vehicles
In order to compare the proposed VTL algorithm with physical traffic light system in SUMO, the $\mathrm{CO}_{2}$ emission of each vehicle is obtained from the simulations.

When the $\mathrm{CO}_{2}$ emission of each vehicle and their average $\mathrm{CO}_{2}$ emission are examined, all vehicles (except SV6 and SV11) using proposed VTL are lower than real traffic light system as shown in Figure 9. On average, the $\mathrm{CO}_{2}$ emission of all vehicles using proposed VTL algorithm is 750278 milligram $(\mathrm{mg})$ and $802120 \mathrm{mg}$ in physical traffic light system. Thus, the proposed VTL algorithm decreases $\mathrm{CO}_{2}$ emission almost $7 \%$ without having any infrastructure like in real traffic light system. The proposed VTL algorithm saves time, in relation to real traffic light system due to the effectiveness of the algorithm and reduces time wasting at the junction. There is no risk yet in the current simulations although the proposed VTL considers not only the vehicles' straight forward directions but also turn left and turn right directions. One of the most significant findings is that the proposed VTL algorithm enables more vehicles to cross the intersection at the same time without collisions.

\section{CONCLUSIONS}

In this study, a VTL algorithm for road intersection was presented. The algorithm is intended for intersections where the deployment of real traffic lights is not costeffective. The proposed VTL algorithm allows more than one vehicle to cross the intersection at the same time without collision by considering various vehicles' directions. The average $\mathrm{CO}_{2}$ emission using proposed VTL algorithm was compared to that of physical traffic light system in SUMO. The algorithm's effectiveness was validated through the implementation of physical traffic light system and virtual traffic light system in SUMO. The simulation results also proved the system's efficiency.

\section{ACKNOWLEDGMENTS}

The author thanks the any anonymous reviewers for helpful comments on the manuscript. I am also grateful to my colleagues for helping in my work. My special thanks go to my Department for allowing me to use the Lab facility.

\section{REFERENCES}

[1] Chen, L., Englund, C.: 'Cooperative intersection management: a survey',IEEE Trans. Intell. Transp. Syst., 2015, PP, (99), pp. 1-17.

[2] A. Bazzi, A. Zanella, and B. M. Masini, "A distributed virtual traffic light algorithm exploiting short range V2V communications, "Ad Hoc Networks, vol. 49, p. 4257,2016

[3] M. Ferreira, R. Fernandes, H. Conceição, W. Viriyasitavat, O.K. Tonguz, "Self- organized traffic control,"7th ACM International Workshop on Vehicular InterNETworking, in: VANET '10, ACM, New York, NY, USA, 2010, p. 85-90.

[4] A . Bazzi, A .Zanella, B. Masini, G. Pasolini, " $A$ distributed algorithm for virtual traffic lights with IEEE 802.11p, "Networks and Communications (EuCNC), 2014 European Conference on, 2014, p. 1-5. 